\title{
Comment On Carbon Production In Deuterium-Metal Systems
}

\author{
DAN CHICEA, Visiting Research Associate Professor, Physics Department, Portland State University (permanent:
} University Lucian Blaga of Sibiu, Physics Department, dan.chicea@ulbsibiu.ro)

\begin{abstract}
Several electrolysis experiments of loading deuterium into different metals foils and thin film films are described. Before and after loading the cathode surface was analyzed by Scanning Electron Microscopy and by Energy Dispersive Spectrometry. High magnification images of the surface of several metals were taken. A control experiment was conducted to make sure that the experimental setup is not accidentally contaminating the samples. A surprising amount of carbon was found on the cathode surface after each D loading experiment. The result is discussed in connection with other experimental results on low energy nuclear transmutations.
\end{abstract}

\section{Introduction}

Starting with March 1989 experimental work was carried on in many laboratories to verify and to reproduce the Fleischman - Pons type experiments [1]. Although most of the effort was focused on excess energy detection, systematic work was carried on to analyze element concentration changes. Many papers reveal significant isotope concentration changes during $\mathrm{H}$ or $\mathrm{D}$ loading experiments in different metals involving different techniques. Some of them, like [2 - 10] are briefly referenced in this paper.

An electrolysis type experiment was designed and performed to achieve a high loading ratio and to analyze the metal surface after deuterium loading. The experimental setup is presented further on.

\section{Experimental setup and control experiment}

The cell consisted of a $50 \mathrm{ml}$ beaker. The cathode holder and the anode were suspended under the rubber cap that covered the beaker. The schematic of the cell is presented in detail in [2]. The anode was a Pt wire, 0.508 $\mathrm{mm}$ diameter, $7 \mathrm{~cm}$ active length, purity $99.997 \%$ and was the same for all the experiments described here. Special care was taken to use pure de-ionized water and clean laboratory Pyrex containers to prepare the electrolyte. The cell cleaning procedure consisted of sinking in nitric acid, rinsing abundantly with de-ionized water and drying with hot air and was applied after each run.

During the entire time span of the experiment preparation, while the cell was open, none of the parts that were put inside was in contact with bare hands or any possible contaminating tool. This was done to avoid contamination both with organic and non-organic substances, that can prevent loading and that can lead to false conclusions.

Energy Dispersive Spectrometry (EDS) analysis, with an accelerating voltage of $20.00 \mathrm{KV}$ and an energy resolution of $61 \mathrm{eV}$, was employed to identify the elements on and under the surface. The same parameters were used for the analysis of all the samples described in this paper. Special care was taken to attach the samples to the sample holder on the SEM machine in such a way that they actually stand outside the sample holder. This prevents false element identification by making sure that the sample holder is relatively far away of the electron beam, thus the elements the sample holder consists of are not present in the X ray spectrum.

For the control experiment the cathode was a Nickel foil $99.9 \%$ purity, having dimensions of $80 \times 30 \mathrm{~mm}$ (in electrolyte), rolled over the Teflon cathode holder. The foil thickness was $0.0127 \mathrm{~mm}$. The electrolyte consisted of $\mathrm{Li}_{2} \mathrm{SO}_{4}, 99.98 \%$ purity in $\mathrm{H}_{2} \mathrm{O}, 1 \mathrm{M}$. A mechanical contact between the platinum wire and the nickel foil was made.

This experiment was conducted first as a control experiment, using a small cathode current density, 0.48 $\mathrm{mA} / \mathrm{cm}^{2}$, and lasted for 142.4 hours. The current density was adjusted to such a small value to allow recombination on the cathode nickel surface. At bigger current density the intense bubbling moves off $\mathrm{D}_{2}$ (or $\mathrm{H}_{2}$ ) and $\mathrm{O}_{2}$ from the cathode surface thus making recombination insignificant [11]. SEM images were taken and EDS analysis was performed before and after the experiment. No significant trace of phosphorous or copper was found on the Nickel foil cathode. If an accidental contamination (cooper or phosphorous for this experiment) occurs or if the purity of the cathode, anode or electrolyte is lower than that indicated by producer, impurities are deposited during electrolysis on cathode surface and are detected by EDS. This experiment revealed that no 
new element was deposited on the cathode surface and this is a proof that the materials used are ultra-pure and that the cell itself, with cathode holder and rubber cap does not have any contamination source.

\section{First experiment of loading $D$ into thin sputtered films}

The same experimental setup was used to perform the deuterium loading experiments. The cathode consisted of quartz slab, having the surface very well cleaned. Over both sides a thin titanium layer, $1200 \AA$ thick and over it a thin layer of Pd, $300 \AA$ thick were deposited by cold sputtering. Both targets used for sputtering had the purity above $99.97 \%$.

A SEM and EDS analyses was not performed on the cathode surface before loading because the targets purity was previously verified and the experiment was started right after sputtering ended.

The electrolyte consisted of $1 \mathrm{M} \mathrm{Li}_{2} \mathrm{SO}_{4}$ in $\mathrm{D}_{2} \mathrm{O}$. The current density was $4.8 \mathrm{~mA} / \mathrm{cm}^{2}$. This experiment lasted for 80 hours. The results of SEM quantitative analysis performed on two different locations on the cathode surface after the experiment are presented in Table I.

\begin{tabular}{|l|c|c|c|}
\hline Sample & Element & Sigma, \% & Atomic concentration, \% \\
\hline Location 1 & $\mathrm{C}$ & 2.65 & 22.96 \\
\cline { 2 - 4 } & $\mathrm{Si}$ & $\mathbf{0 . 9 6}$ & $\mathbf{3 7 . 8 2}$ \\
\cline { 2 - 4 } & $\mathrm{Ti}$ & $\mathbf{0 . 9 7}$ & $\mathbf{2 1 . 8 4}$ \\
\cline { 2 - 4 } & $\mathrm{Cu}$ & $\mathbf{0 . 8}$ & $\mathbf{1 4 . 9 0}$ \\
\cline { 2 - 4 } & $\mathrm{Pd}$ & $\mathbf{0 . 6 0}$ & 2.75 \\
\hline Location 2 & $\mathrm{C}$ & 2.51 & 29.18 \\
\cline { 2 - 4 } & $\mathrm{Si}$ & $\mathbf{1 . 0 3}$ & $\mathbf{3 8 . 5 6}$ \\
\cline { 2 - 4 } & $\mathrm{Ti}$ & $\mathbf{1 . 0 2}$ & $\mathbf{2 1 . 6 9}$ \\
\cline { 2 - 4 } & $\mathrm{Cu}$ & $\mathbf{0 . 8 1}$ & $\mathbf{8 . 0 7}$ \\
\cline { 2 - 4 } & $\mathrm{Pd}$ & $\mathbf{0 . 6 0}$ & $\mathbf{2 . 5 0}$ \\
\hline
\end{tabular}

Table I - Results of the SEM analysis on the first Pd/Ti thin film cathode.

A SEM image of the cathode after electrolysis is presented in Figure 1. It reveals that the surface presents a granulated aspect, which proves that this cathode absorbed Deuterium in big amount. The mechanical stress produced by the different expansion of the two metals caused by D absorption produced micro-cracks on the surface. One of them is presented in Figure 1.

\section{The second experiment of loading D into thin sputtered films}

The same experiment was repeated. The cathode consisted of quartz slab and over both sides a $1100 \AA$ thick thin titanium layer and over it a thin layer of Pd, $200 \AA$ thick were deposited by cold sputtering. The same targets were used for sputtering and they had a purity above $99.97 \%$. The same type of electrolyte, consisting of $1 \mathrm{M}$ $\mathrm{Li}_{2} \mathrm{SO}_{4}$ in $\mathrm{D}_{2} \mathrm{O}$ was used. The current density was $5 \mathrm{~mA} / \mathrm{cm}^{2}$. This experiment lasted for 160 hours. The results of SEM quantitative analysis performed on a spectrum acquired in the map mode after deuterium loading are presented in Table II. An SEM-EDS analysis on the cathode surface before experiment was not preformed for the same reason as for the previous experiment. 


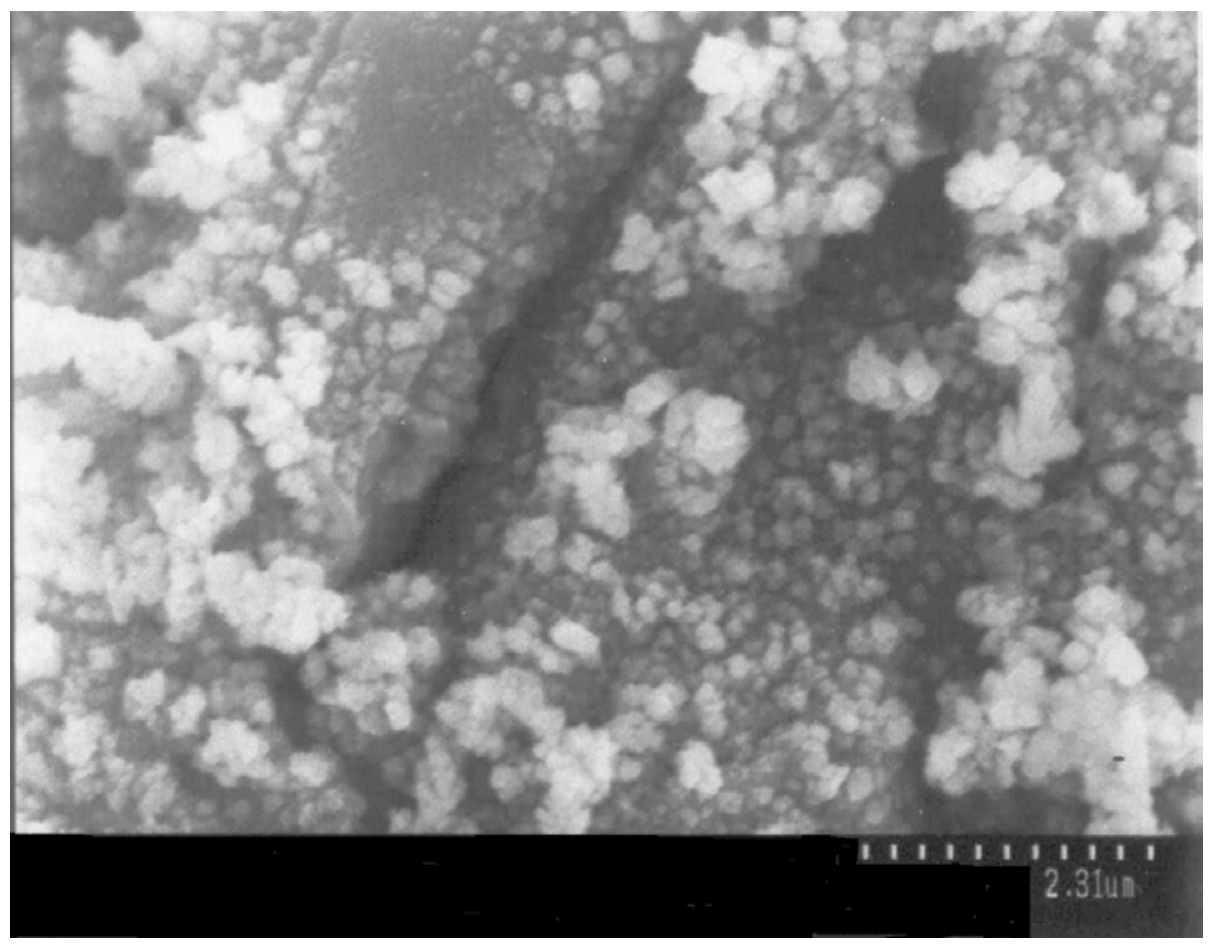

Figure 1: The SEM image of the first sputtered Ti/Pd thin film cathode after D loading

Examining the surface of the cathode presented in Figure 2 we notice that it has fissures, which is evidence of deuterium loading. The surface is granulated and also presents deep holes and apparently parts of the palladium thin layer have been completely detached apart, in the upper right corner.

\begin{tabular}{|c|c|c|}
\hline Element & Sigma, \% & Element concentration, \% \\
\hline $\mathrm{C}$ & 2.65 & 25.13 \\
\hline $\mathrm{Si}$ & 0.96 & 35.62 \\
\hline $\mathrm{Ti}$ & 0.97 & 24.84 \\
\hline $\mathrm{Cu}$ & 1.08 & 12.67 \\
\hline $\mathrm{Pd}$ & 0.6 & 1.74 \\
\hline
\end{tabular}

Table II - Results of the SEM analysis on the second Pd/Ti thin film cathode.

An image of the surface of the cathode after the second experiment of loading deuterium into titaniumpalladium thin films cathode is presented in Figure 2. 


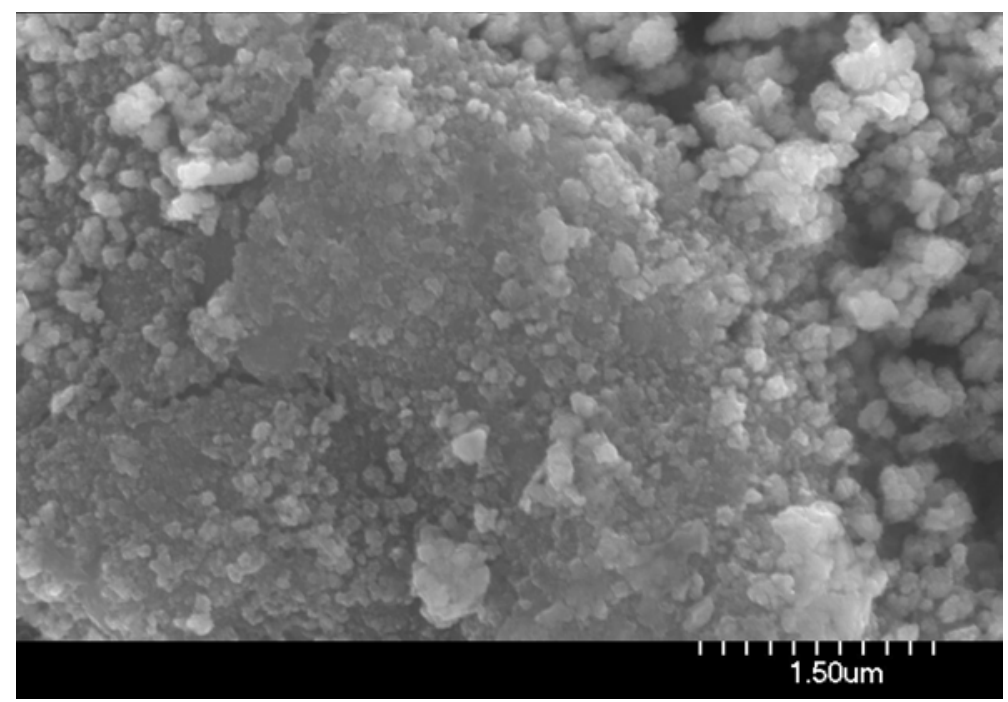

Figure 2 - SEM image of the second sputtered Ti/Pd thin film cathode after D loading

Examining the element concentration results presented in Tables I and II we notice that among titanium and palladium that were sputtered on the quartz slab carbon, silicon and copper were found. The silicon amount that was detected comes from the composition of the quartz substrate. As teflon does not get dissolved in the electrolyte this can not be the explanation for the amount of carbon that was found. Unlike for the control experiment and for the experiment described in the next section of this paper, where a mechanical contact between the cathode foil and the platinum wire was made, a small copper tape was attached on the quartz slide before sputtering and on it a small rectangle was masked with adhesive tape. The layers were sputtered over the whole slide and after sputtering the adhesive tape was removed. The clean rectangular shape copper surface was used to attach the cathode electrical connector to the cathode. The contact was insulated with silicone adhesive. There might be a chance that the insulation ceased at a certain time on a small portion and that the electrolyte got in contact with the copper tape underneath, dissolving part it. The dissolved copper might have been electrodeposited on the cathode surface. This may be the explanation of the copper found after this experiment but not found after the control experiment and after the experiment presented in the next section

\section{Experiment of loading D into thin Palladium foil}

Another experiment was completed using the same experimental setup as the control experiment. A Palladium foil $99.9 \%$ purity, with the thickness of $0.025 \mathrm{~mm}$ was used as cathode. Before the electrolysis the palladium foil was thoroughly cleaned using acetone to remove any possible organic compounds and then the same procedure described in section 2. The electrolyte consisted of $\mathrm{Li}_{2} \mathrm{SO}_{4}$ in $\mathrm{D}_{2} \mathrm{O}, 0.5 \mathrm{M}$. Before the electrolysis an $\mathrm{X}$ ray spectrum was acquired and EDS analysis was performed. No element other than palladium was found in a concentration above two standard deviations.

The experiment performed with this cathode was conducted with a higher current density, $7.5 \mathrm{~mA} / \mathrm{cm}^{2}$ and lasted for 45 hours. The results of EDS quantitative analysis performed on the cathode after the experiment are presented in Table III.

\begin{tabular}{|c|c|}
\hline Element & Atomic concentration, \% \\
\hline $\mathbf{C}$ & $\mathbf{2 3 . 6 8}$ \\
\hline $\mathbf{P d}$ & $\mathbf{7 6 . 3 2}$ \\
\hline
\end{tabular}

Table III - Results of the SEM analysis on Pd thin foil cathode.

\section{Discussion}

The results presented in this paper, taken alone, appear to be surprising, but they should be viewed in the context of other papers written on this subject. In [2] copper was found after loading hydrogen into high purity 
nickel foil. In reference [3], calcium was found in electrolyte and after repeating the experiment tritium was found in the electrolyte as well [4]. References [5] and [6] deal with Palladium loaded with Deuterium. Titanium and calcium was found in [5]. EDX revealed the formation of zinc in the palladium cathode that was subject to a 2 atm $\mathrm{D}_{2}$ gas loading procedure, in [6]. In [7] both a titanium and a palladium cathode were loaded with deuterium. SEM and EDS were employed in analyzing the samples and elements like V, Cr, Fe, Ni and Zn were found to be produced during the experiments.

Other work, like [8], reported a 270 hours experiment of loading hydrogen and deuterium into palladium and found that the amount of carbon actually decreased, but they provide no explanation for the provenience of carbon on palladium cathodes. [9] Describes a 220 hours experiment of loading deuterium into palladium. Large values for carbon and oxygen, platinum, lead, zinc, chromium and in some cases copper, calcium, magnesium and sodium were found. [10] Presents a gas loading experiment that produces carbon on the palladium electrodes that were used for sparking.

The experiments presented in this work reveal that when titanium, palladium or a combination of them was loaded with deuterium a considerable amount of carbon was found on the surface of the cathode when the current density was adjusted in such a manner that deuterium was loaded. These results suggest that there is a strong correlation between achieving a high loading ratio and the appearance of new elements on the cathode.

The appearance of carbon on palladium after being loaded with deuterium might be the result of low energy nuclear reactions undergoing in a novel way. The multi-body fusion of D-s is a process that is very improbable in vacuum but can become significant at high $\mathrm{D}$ density, caused by a strong confinement inside the palladium or titanium lattice and in the presence of an increased "free" electron concentration.

The deuterium loading experiments and concentration analysis described in this paper fit well in the experimental landscape consisting of the results briefly reviewed here and of many others as important as these, but not mentioned in this paper. Element concentration changes after deuterium loading experiments in different metals are intriguing, by the lack of a satisfactory conventional explanation. Finding an explanation for the changes keeps being a challenge for the theoretical physics.

\section{Acknowledgements}

I fully acknowledge Dr. George H. Miley and Dr. Edmund K. Storms for the fruitful discussions and good advice.

\section{References}

1. M. Fleischman, and S. Pons, "Electrochemically Induced Nuclear Fusion of Deuterium," J. Electroanal. Chem., pp.261-301, 1989.

2. D. Chicea, Comment on S.E.M. Analysis Results of Post-Hydrogen Absorption Nickel Surfaces, Journal of New Energy, Vol. Nr. March, 2003.

3. R. Notoya, Cold Fusion by Electrolysis in a Light Water Potassium Carbonate Solution With a Nickel Electrode, Fusion Technology vol. 24, p. 202, 1993.

4. R. Notoya, Y. Noya, T. Ohnishi, Tritium Generation and Large Excess Heat Evolution by Electrolysis in Light and Heavy Water Potassium Carbonate Solution with a Nickel Electrode, Fusion Technology vol. 26, pp. 179, 1994.

5. Y. Iwamura, T. Itoh, N. Gotoh, I. Toyoda, Detection of Anomalous Elements, X-rays, and Excess Heat in a $\mathrm{D}_{2}$-Pd System and its Interpretation by the Electron Induced Nuclear Reaction Model, Fusion Technology vol. 33, pp. 476, 1998.

6. V. Nassisi, Transmutation of Elements in Saturated Palladium Hydrides by an XeCl Excimer Laser, Fusion Technology vol. 33, pp. 468, 1998.

7. J. Dash, R. Kopecec, S. Miguet, Excess Heat and Unexpected Elements from Aqueous Electrolysis with Titanium and Palladium Cathodes, Proc. Of the $32^{\text {nd }}$ Intersociety Conversion Energy Conference, vol. 2, pp. 1350-1355, 1997.

8. Divisek J, Fuerst L, Balej J; Energy balance of D2O electrolysis with a palladium cathode. Part II. Experimental results J. Electroanal. Chem. 278, pp. 99, 1989.

9. Ulman M, Liu J, Augustynski J, Meli F, Schlapbach L; Surface and electrochemical characterization of Pd cathodes after prolonged charging in LiOD + D2O solutions J. Electroanal. Chem. 286, 257, 1990.

10. Yamada H, Fujiwara T; Neutron emission from palladium point electrode in pressurized deuterium, gas under DV voltage application Int. J. Soc. Mat. Eng. Resources 6(1), pp. 14, 1998. 
11. Storms Ed., A Critical Evaluation of the Pons-Fleischmann Effect, Infinite Energy, Vol. 6, Issue no. 31, pp. $10,2000$. 